\title{
Arithmetical transfinite induction and hierarchies of functions
}

\author{
by
}

Zygmunt Ratajczyk (Warszawa)

\begin{abstract}
We generalize to the case of arithmetical transfinite induction the following three theorems for PA: the Wainer Theorem, the Paris-Harrington Theorem, and a version of the Solovay-Ketonen Theorem. We give uniform proofs using combinatorial constructions.
\end{abstract}

1. Introduction. The results of this paper are concerned with the theory of the arithmetical transfinite induction $I\left(\varepsilon_{\alpha}\right)$ (also denoted in the literature by $T I\left(\varepsilon_{\alpha}\right) ; \varepsilon_{\alpha}$ is a recursive number). We generalize to the case of $I\left(\varepsilon_{\alpha}\right)$ the following three theorems for mathematical induction, i.e. for the theory of Peano Arithmetic (PA):

1.1. TheOREM (Wainer [11]). If $\varphi(x, y) \in \Sigma_{1}$ and $\mathrm{PA} \vdash \forall x \exists y \varphi(x, y)$, then there exists an $\alpha<\varepsilon_{0}$ such that $N \vDash \forall x \exists y<H_{\alpha}(x) \varphi(x, y)$.

Here $H_{\alpha}: \alpha<\varepsilon_{0}$ denotes the usual Hardy hierarchy of functions.

Let $R\left(T ; \Sigma_{n}\right)$ denote the following sentence in $L_{\mathrm{PA}}$ :

$$
\forall \theta(x) \in \Sigma_{n} \forall x\left[T \vdash \theta(\underline{x}) \rightarrow \operatorname{Tr}_{\Sigma_{n}}(\theta(x))\right],
$$

where $\underline{x}$ is the term, called numeral, having value $x$. This sentence is referred to as the uniform reflection principle for $\Sigma_{n}$-formulas over $T$.

Next, let $W(H, \ldots)$ be a property of finite sets $H$. Then $X \rightarrow(W)_{k}^{n}$ is the combinatorial property

$$
\forall P:[X]^{n} \rightarrow k \exists H \subseteq X\left[W(H) \wedge\left|P\left([H]^{n}\right)\right|=1\right],
$$

where $[X]^{n}$ is the set of all $n$-element subsets of the set $X$. The combinatorial principle

$$
\forall n, k, r \exists M\left[M \rightarrow(\min H<|H| \wedge r<|H|)_{k}^{n}\right]
$$

is denoted by $\mathrm{PH}$.

1.2. Theorem (Paris-Harrington $[6]$ ). $\mathrm{PA} \vdash\left[\mathrm{PH} \Leftrightarrow R\left(\mathrm{PA} ; \Sigma_{1}\right)\right]$. 
1.3. Theorem (Solovay-Ketonen [3]). PA $\vdash\left[\mathrm{PH} \Leftrightarrow \forall x \exists y H_{\varepsilon_{0}}(x)=y\right]$.

A generalization of Theorem 1.1 can be symbolically written as follows:

1.4. THEOREM. If $\varphi(x, y) \in \Sigma_{1}$ and $I\left(\varepsilon_{\alpha}\right) \vdash \forall x \exists y \varphi(x, y)$ then there exists a $\beta<\varepsilon_{\alpha+1}$ such that $N \vDash \forall x \exists y<H_{\beta}(x) \varphi(x, y)$.

The exact meaning of this theorem depends on the character of fundamental sequences which are used to extend the Hardy hierarchy $H_{\beta}(x)$ to $\beta<\varepsilon_{\alpha+1}$. In Ratajczyk [8, Section III.1] the above theorem was proved under the assumption that the fundamental sequences form a $\left(B^{+}\right)^{2}-\varepsilon$-system, a notion recalled here in 1.7 ; such systems are a generalization of built-up systems considered by Schmidt [10]. The exact formulation of our generalizations of Theorems 1.1-1.3 will be preceded by recalling some notions and definitions from [8]. Now we only want to say that the generalization of 1.4 proved in this paper is not essentially different from that in [8]. But the proof is shorter and relies on another idea.

Let $\lambda$ be a primitive recursive ordinal number. We identify $\lambda$ with an ordering $<_{\lambda} \in$ Prec having type $\lambda$ and domain $N$ or rather with a $\Sigma_{1}$-formula defining this ordering in PA.

We say that $\lambda$ is an $\varepsilon$-system of notations in PA of class $\Sigma_{1}$ if

(i) some additional $\Sigma_{1}$-formulas are given, defining in PA the operations on $\lambda$ denoted by $\alpha+\beta, \omega^{\alpha}, \varepsilon_{\beta}$ and defining the set Lim, and

(ii) some axiomatically described properties of these operations are provable in PA, which enable us to infer in PA all basic properties of these operations, e.g. PA $\vdash \omega^{\varepsilon_{\alpha}}=\varepsilon_{\alpha}, \mathrm{PA} \vdash\left[\alpha<\varepsilon_{\beta} \rightarrow \omega^{\alpha}<\varepsilon_{\beta}\right]$, PA $\vdash[\alpha \in \operatorname{Lim} \rightarrow$ $\left.\omega^{\alpha}=\lim _{\beta<\alpha} \omega^{\beta}\right]$, etc.

The details can be found in [8, Section I.1], in fact only for $I \Sigma_{1}$, but we can relativize them all to $\mathrm{PA}$.

The following definition can also be found there:

1.5. Definition. We say that $P(x, y)=z \in L_{\mathrm{PA}}$ defines in $\mathrm{PA}$ a $B^{+}$- $\varepsilon$-system of sequences for $\lambda$ if and only if PA proves that for all $\alpha, \beta, \gamma<\lambda$ and for all $n$

(1) $\quad P$ is a $B^{+}$-system of sequences, i.e. $\alpha \in \operatorname{Lim} \rightarrow \alpha_{n} \nearrow \alpha$, where $\alpha_{n}=P(\alpha, n)$, and $\alpha_{n}+1<\beta<\alpha \rightarrow \alpha_{n}+1 \leq \beta_{0}<\alpha$,

(2) $\quad(\alpha+\beta)_{n}=\alpha+\beta_{n}$ if the last component of the Cantor normal representation of $\alpha$ is greater than or equal to the first component of $\beta$,

(3) $\left(\omega^{\alpha+1}\right)_{n}=\omega^{\alpha}(n+1)+1$,

(4) $\left(\omega^{\alpha}\right)_{n}=\omega^{\alpha_{n}}$ for $\alpha \in \operatorname{Lim}, \alpha \neq 0$ and not of the form $\varepsilon_{\beta}$,

(5) $\left.\quad\left(\varepsilon_{0}\right)_{n}=\omega^{\omega^{\cdot \cdot \omega}}\right\} n+1$ times, 
(6) $\quad\left(\varepsilon_{\beta+1}\right)_{n}=\omega_{n+1}^{\varepsilon_{\beta}+1}$, where in general $\omega_{0}^{\gamma}=\gamma, \omega_{n+1}^{\gamma}=\omega^{\omega_{n}^{\gamma}}$.

1.6. N ot e. One can show the following (for the proof see [8], Section I.2):

For each $\varepsilon$-system of notations $\lambda$ in PA of class $\Sigma_{1}$ there exists $P(\alpha, n)=$ $\beta \in L_{\mathrm{PA}}$ of class $\Sigma_{1}$ which defines a $B^{+}-\varepsilon$-system for $\lambda$ in PA.

For concrete primitive recursive ordinals, e.g. for $\varepsilon_{0}$ and $\Gamma_{0}$, there exist some natural $B^{+}-\varepsilon$-systems.

For $\lambda=\varepsilon_{0}$ the system $P(\alpha, n)$ is uniquely determined by (2)-(5) of 1.5 , and one can show that it is a $B^{+}$-system of sequences. The standard system of sequences for numbers $<\varepsilon_{0}$, i.e. with $\left(\omega^{\alpha+1}\right)_{n}=\omega^{\alpha} \cdot n$, satisfies the condition: $\alpha_{n}<\beta<\alpha$ implies $\alpha_{n} \leq \beta_{0}<\alpha$ for all $\alpha, \beta$, which is characteristic for built-up sequences of Schmidt [10] .

The system of fundamental sequences for the Feferman number $\Gamma_{0}$ appearing in [10] satisfies the same condition. Moreover, it satisfies conditions very similar to $(2)-(6)$ for $(\alpha+\beta)_{n},\left(\omega^{\alpha}\right)_{n},\left(\varepsilon_{\alpha}\right)_{n}$, and some natural inductive condition for the remaining operations building $\Gamma_{0}$. The system $\left(\alpha_{n}\right)_{n \in \omega}$ for $\alpha<\Gamma_{0}$ defined in accordance with (2)-(6) is also natural and can be shown to be a $B^{+}-\varepsilon$-system.

In this paper we only use the following systems of sequences:

1.7. Definition. A system $P$ of sequences for $\lambda$ is called a $\left(B^{+}\right)^{2}-\varepsilon$ system of sequences in PA iff PA proves:

(1) $P$ is a $B^{+}-\varepsilon$-system,

(2) $\quad\left(\varepsilon_{\alpha}\right)_{n}=\varepsilon_{\alpha_{n}^{\prime}}$ for $\alpha \in \operatorname{Lim}, \alpha>0$, and $n>0$, where

(3) $\quad \alpha_{n}^{\prime}$ is a $B^{+}$-like system, i.e. $\alpha_{n}^{\prime}+1<\beta<\alpha$ implies that $\alpha_{n}^{\prime}+1 \leq$ $\beta_{0}^{\prime}<\alpha$.

One can check that for limit $\alpha, 0<\alpha<\Gamma_{0}$, the system of [10] has the property that $\left(\varepsilon_{\alpha}\right)_{n}=\varepsilon_{\alpha_{n-1}}$ for $n>0$ and $\alpha$ such that $\alpha \neq \varepsilon_{\alpha}$. Hence this system of sequences for $\Gamma_{0}$, which satisfies $(1.5)(2)-(6)$, is a $\left(B^{+}\right)^{2}-\varepsilon$-system.

Throughout the paper we assume that if $\lambda$ is given then a $\left(B^{+}\right)^{2}$ - $\varepsilon$-system $P$ of sequences for $\lambda$ is fixed.

The next definition makes precise the intuitive notion of a "Hardy type iteration". Assume that PA $\vdash$ " $G$ is a function"; it is not assumed that PA proves that the formula $G$ defines a total function.

1.8. To the intuitive notion of Hardy iteration of $G$ there corresponds a formula in PA which defines in PA a sequence $G_{\alpha}: \alpha<\lambda$ about which PA proves:

(1) $G_{0}=\mathrm{id}$ (is total),

(2) $G_{\alpha+1}(x) \simeq G_{\alpha}(G(x))$,

(3) $G_{\alpha}(x) \simeq G_{\alpha_{x}}(x)$, 
(4) $\quad G_{\alpha}(x) \downarrow$ (is defined) $\Leftrightarrow$ using (1)-(3) as some reductive operations we can compute $G_{\alpha}(x)$ in a finite number of steps.

Here $\simeq$ is equality for partial functions. Of course such a formula $G_{\alpha}(x)=y$ always exists.

Next we define some formal theories of Hardy hierarchies. Let $H^{0}(x)=$ $x+1$ (denoted also by $H(x)$ ). For $n>0$, let $H^{n}$ denote the function in PA (or rather in the extension of PA by the definition of $H^{n}$ ) defined in the following way:

$$
\begin{aligned}
H^{n}(a)=\min _{b}\left\{\forall \theta<a \forall \bar{a}<a\left[\theta \in \Pi_{n-1} \wedge\right.\right. & \operatorname{Tr}_{\Sigma_{n}}(\exists \bar{y} \theta(\bar{a}, \bar{y})) \\
& \left.\left.\rightarrow \exists \bar{b}<b \operatorname{Tr}_{\Pi_{n-1}}(\theta(\bar{a}, \bar{b}))\right]\right\},
\end{aligned}
$$

where $\bar{a}<a$ means that all terms of the sequence $\bar{a}$ are $<a$, and $\operatorname{Tr}_{\Sigma_{n}}$ denotes the standard $\Sigma_{n}$ truth formula for all $\Sigma_{n}$-formulas.

\subsection{Definition.}

$$
\begin{gathered}
T H^{0}\left(<\varepsilon_{\alpha}\right) \equiv \mathrm{PA} \cap \Pi_{2}+\left\{\forall x \exists y\left[H_{\underline{\beta}}(x)=y\right]: \beta<\varepsilon_{\alpha}\right\}, \\
T H\left(\varepsilon_{\alpha}\right) \equiv \mathrm{PA} \cup\left\{\forall x \exists y\left[\left(H^{n}\right)_{\varepsilon_{\underline{\alpha}}}(x)=y\right]: n \in \omega\right\} .
\end{gathered}
$$

Moreover, $I\left(<\varepsilon_{\alpha}\right)$ denotes the sum of the theories $I(\beta)$ for $\beta<\varepsilon_{\alpha}$.

The theories $T H^{0}\left(<\varepsilon_{\alpha}\right)$ and $T H\left(\varepsilon_{\alpha}\right)$ depend in fact on the choice of the system of sequences $P$, but this is not important because $P$ is fixed. One can show that $T H^{0}\left(<\varepsilon_{\alpha+1}\right) \equiv P-T H^{0}\left(<\varepsilon_{\alpha+1}\right)$, where the last notation comes from [8, III.1].

Now we have at our disposal all notions which are necessary to formulate the announced generalizations of Theorems 1.1-1.3. But first let us mention the following well-known generalization of Theorem 1.1.

$$
\text { 1.10. } \mathrm{PA} \cap \Pi_{2} \equiv T H^{0}\left(<\varepsilon_{0}\right) \text {. }
$$

For the proof see Hájek and Paris [2] or Ratajczyk [7]. Assume now that $\lambda$ is an arbitrary $\varepsilon$-system of notations in PA, $\varepsilon_{\alpha+1}<\lambda$ and there is given a system of sequences for $\lambda$ (by convention, necessarily a $\left(B^{+}\right)^{2}$ - $\varepsilon$-system). The theorem which we will prove is in fact a further generalization of 1.1.

1.11. TheOREM. If the system of sequences $P$ is of class $\Sigma_{1}$ then

$$
I\left(\varepsilon_{\alpha}\right) \cap \Pi_{2} \equiv T H^{0}\left(<\varepsilon_{\alpha+1}\right) .
$$

The proof is based on the following fundamental equivalence:

1.12. TheOREM (PA). $I\left(\varepsilon_{\alpha}\right) \equiv T H\left(\varepsilon_{\alpha}\right)$ for $\alpha<\lambda$.

Theorem 1.11 was also proved in [8], similarly to 1.4. The proof presented here relies on another idea using the "reflexivity of the hierarchy $T H\left(\varepsilon_{\alpha}\right)$ ": 
1.13. Lemma. PA proves that for every $\alpha<\lambda$ and for every $k \in \omega$, $T H\left(\varepsilon_{\alpha}\right) \vdash R\left(\bigcup_{n} T H\left(\left(\varepsilon_{\underline{\alpha}}\right)_{n} ; \Sigma_{k}\right)\right)$.

Then we prove that the reflexivity of $T H\left(\varepsilon_{\alpha}\right): \alpha<\lambda$ implies that $I\left(\varepsilon_{\alpha}\right) \subseteq T H\left(\varepsilon_{\alpha}\right)$; the inclusion $T H\left(\varepsilon_{\alpha}\right) \subseteq I\left(\varepsilon_{\alpha}\right)$ is obvious. The presented idea is the same as those used by Schmerl to prove that $I\left(\varepsilon_{\alpha}\right)$ is included in a theory of $\alpha$-fold iteration of the reflection principle. The essential difficulty now is to prove the above lemma, which is done by combinatorial means.

Now we describe our generalizations of the other two theorems, i.e. of Theorems 1.2 and 1.3. Let

$$
\begin{array}{ll}
\overline{\mathrm{PH}}_{\alpha} \Leftrightarrow \forall x \exists y\left([1, y] \rightarrow\left(H \text { is }\left(\varepsilon_{\alpha}\right)_{x} \text {-large } \wedge|H| \geq x+1\right)_{x}^{x}\right) & \text { if } \alpha \in \operatorname{Lim}, \\
\overline{\mathrm{PH}}_{\alpha} \Leftrightarrow \forall x \forall y \exists z\left([y, z] \rightarrow\left(H \text { is } \varepsilon_{\beta} \text {-large } \wedge|H| \geq x+1\right)_{x}^{x}\right) & \text { if } \alpha=\beta+1,
\end{array}
$$

where by convention $\varepsilon_{-1}=\omega$. This principle is an easy generalization of the principle of Paris and Harrington $\mathrm{PH}\left(\mathrm{PH} \equiv \overline{\mathrm{PH}}_{0}\right)$. Another generalization of $\mathrm{PH}$, denoted by $\mathrm{PH}_{\alpha}$, was introduced in [8]. It expresses the property of $\alpha$-fold iteration of the principle $\mathrm{PH}$. It was proved in [8] that $\mathrm{PH}_{\alpha}$ is equivalent in PA to $R\left(I\left(<\varepsilon_{\alpha}\right) ; \Sigma_{1}\right)$ and, in consequence, is independent of PA. Here we prove the same for $\overline{\mathrm{PH}}_{\alpha}$.

1.14. TheOREM. If the system of sequences $P$ is of class $\Sigma_{1}$ then

$$
\mathrm{PA} \vdash \forall \alpha<\lambda\left[\overline{\mathrm{PH}}_{\alpha} \Leftrightarrow R\left(I\left(<\varepsilon_{\alpha}\right) ; \Sigma_{1}\right)\right] .
$$

1.15. TheOREM. If the system of sequences $P$ is of class $\Sigma_{1}$ then

$$
\mathrm{PA} \vdash \forall \alpha<\lambda\left[\forall x \exists y\left(H_{\varepsilon_{\alpha}}(x)=y\right) \Leftrightarrow R\left(I\left(<\varepsilon_{\alpha}\right) ; \Sigma_{1}\right)\right] .
$$

Now we describe in more detail the differences between $[8]$ and this paper.

The main aim of this paper as well as of [8] is to provide different applications of the notion of an $a$-skeleton (see further Def. 3.3). This notion and its properties (and generalizations) form a uniform basis for the proofs of Theorems 1.11-1.15. But a new idea used here in the proof of Theorems 1.11 and 1.12 does not require iterations of the construction of $a$-skeletons. As a result we obtain a new and simpler proof of the generalization of the Wainer Theorem to the case of $I\left(\varepsilon_{\alpha}\right)$.

Since we use here most of the basic notions of [8], the present paper may be used as an introduction to the long paper [8].

We will systematically use the basic technical results of [8], which we explicitly formulate. The present paper can be read independently of $[8]$ if the reader is not interested in the proofs of all technical results.

2. Derivation of 1.12. In this section, using Lemma 1.13 we prove Theorem 1.12. Since the inclusion $T H\left(\varepsilon_{\alpha}\right) \subseteq I\left(\varepsilon_{\alpha}\right)$ in 1.12 is obvious, it remains to show that $\mathrm{PA} \vdash \forall \alpha<\lambda I\left(\varepsilon_{\alpha}\right) \subseteq T H\left(\varepsilon_{\alpha}\right)$. 
In the proof we use the following

LEMMA (Schmerl [9]). Let $\varphi(x) \in L_{\mathrm{PA}}$. If

$$
\mathrm{PA} \vdash \forall \alpha<\lambda[(\mathrm{PA} \vdash\ulcorner\forall \beta<\underline{\alpha} \varphi(\beta)\urcorner) \rightarrow \varphi(\alpha)],
$$

then $\mathrm{PA} \vdash \forall \alpha<\lambda \varphi(\alpha)$.

Proof of Theorem 1.12. Put $\varphi(\alpha):=I\left(\varepsilon_{\alpha}\right) \subseteq T H\left(\varepsilon_{\alpha}\right)$. In view of the Lemma it is enough to show that

$\mathrm{PA} \vdash \forall \alpha<\lambda\left[\left(\mathrm{PA} \vdash\left\ulcorner\forall \beta<\underline{\alpha} I\left(\varepsilon_{\beta}\right) \subseteq T H\left(\varepsilon_{\beta}\right)\right\urcorner\right) \rightarrow I\left(\varepsilon_{\alpha}\right) \subseteq T H\left(\varepsilon_{\alpha}\right)\right]$.

We show this statement working in PA.

We fix $\alpha<\lambda$ and assume that PA $\vdash\left\ulcorner\forall \beta<\underline{\alpha} I\left(\varepsilon_{\beta}\right) \subseteq T H\left(\varepsilon_{\beta}\right)\right\urcorner$. Hence it follows in particular that

$$
\begin{aligned}
& \mathrm{PA} \vdash\left\ulcorner\forall n I\left(\left(\varepsilon_{\underline{\alpha}}\right)_{n}\right) \subseteq T H\left(\left(\varepsilon_{\underline{\alpha}}\right)_{n}\right)\right\urcorner, \quad \text { i.e. } \\
& \mathrm{PA} \vdash\left\ulcorner\bigcup_{n} I\left(\left(\varepsilon_{\underline{\alpha}}\right)_{n}\right) \subseteq \bigcup_{n} T H\left(\left(\varepsilon_{\underline{\alpha}}\right)_{n}\right)\right\urcorner, \quad \text { i.e. } \\
& \left.\mathrm{PA} \vdash\left\ulcorner I\left(<\varepsilon_{\underline{\alpha}}\right)\right) \subseteq \bigcup_{n} T H\left(\left(\varepsilon_{\underline{\alpha}}\right)_{n}\right)\right\urcorner .
\end{aligned}
$$

Hence we deduce by 1.13 that $T H\left(\varepsilon_{\alpha}\right) \vdash R\left(I\left(<\varepsilon_{\underline{\alpha}}\right) ; \Sigma_{k}\right)$ for all $k$.

The old result of Gentzen [1] says that $I\left(\varepsilon_{\alpha}\right)$ is a consequence of the full uniform reflection principle over $I\left(<\varepsilon_{\alpha}\right)$. Hence $T H\left(\varepsilon_{\alpha}\right) \vdash I\left(\varepsilon_{\alpha}\right)$, i.e. $I\left(\varepsilon_{\alpha}\right) \subseteq T H\left(\varepsilon_{\alpha}\right)$, which was to be proved.

3. Technical results. In this section we collect almost all technical results which we use later, in particular those which are needed to prove the key Lemma 1.13.

We first formulate the basic properties of $\left(B^{+}\right)^{2}-\varepsilon$-systems of sequences and the Hardy hierarchy $H_{\beta}$.

We write $\alpha \Rightarrow_{x} \beta$ if there exists a finite sequence $\alpha=\alpha^{0}, \ldots, \alpha^{l}=\beta$ such that $\left(\alpha^{i}\right)_{x}=\alpha^{i+1}$ for all $i$ with $0 \leq i<l$ (we define $\alpha \Rightarrow \leq x \beta$ analogously). We call a set $A$-large iff $\left(S^{A}\right)_{\alpha}(\min A) \downarrow$, where $\operatorname{dm} S^{A}=A \backslash\{\max A\}$ and $S^{A}(a)=\min \{b \in A: a<b\}$ for $a \in \operatorname{dm} S^{A}$.

3.1. Lemma (PA). For every limit $\alpha<\lambda$

$$
\begin{gathered}
\forall n\left(\alpha_{n+1} \Rightarrow_{0} 0 \rightarrow \alpha_{n+1} \Rightarrow_{0} \alpha_{n}+1\right), \\
\forall n\left[\left(\varepsilon_{\alpha}\right)_{n+1} \Rightarrow_{0} 0 \rightarrow\left(\varepsilon_{\alpha}\right)_{n+1} \Rightarrow_{0} \varepsilon_{\alpha_{n}^{\prime}+1}\right],
\end{gathered}
$$

where $\alpha_{n}^{\prime}$ is the number from the definition of $\left(B^{+}\right)^{2}-\varepsilon$-systems.

Moreover, for every increasing function $G$ satisfying $\forall x \in \operatorname{dm} G[0<$ $x<G(x)]$ we have:

(3) For every natural number $d$ the set $X_{d}=\left\{\alpha<\lambda: \exists x \geq 1 G_{\alpha}(x)\right.$ $\leq d\}$ is finite and the implication $\alpha \in X_{d} \rightarrow \alpha_{0} \in X_{d}$ is true (in consequence, if $\alpha \in X_{d}$ then $\left.\alpha \Rightarrow_{0} 0\right)$,

(4) $G_{\alpha}$ is increasing, i.e. $G_{\alpha}(y) \downarrow \wedge x \leq y \rightarrow G_{\alpha}(x) \leq G_{\alpha}(y)$. 
(5) $\quad G_{\alpha_{y}}(x)<G_{\alpha}(x)$ for all $0 \leq y<x$, i.e. the hierarchy $G_{\alpha}$ is locally increasing in the terminology of [8].

(6) If a function $F$ is bounded by $G$, i.e. if $\forall x \in \operatorname{dm} F[G(x) \downarrow \rightarrow F(x) \leq$ $G(x)$, then the function $F_{\alpha}$ is bounded by $G_{\alpha}$.

(7) If $\alpha \Rightarrow_{x} \beta$ then for every finite set $A$ with $x \leq \min A$ the following implication is true: $A$ is $\alpha$-large $\rightarrow A$ is $\beta$-large.

(8) If the last component of the Cantor normal representation of $\alpha$ is greater than or equal to the first component of $\beta$ then $G_{\alpha+\beta} \simeq G_{\alpha} \circ G_{\beta}$.

Proof. Assertions (3), (4), (5), (7) were proved in [8] for $B^{+}$- $\varepsilon$-systems (see Chapter I.2). (1), (2) are immediate consequences of the definition of $B^{+}$-systems and $\left(B^{+}\right)^{2}-\varepsilon$-systems. Moreover, observe that $(7)$ is an immediate consequence of (5). Hence it remains to show (6) and (8) but for the sake of completeness we also show (4) and give a sketch of proof of (5) for $B^{+}$- - -systems.

Proof of (4) and (5). Let $X_{d}=\left\{\alpha: \exists x \geq 1 G_{\alpha}(x) \leq d\right\}$. We show by induction on the finite set $<\uparrow X_{d}$ that (4) and (5) are true for all $\alpha$, and for $x$ such that $G_{\alpha}(x) \leq d$. We only exhibit the inductive step for limit $\alpha \in$ $X_{d}$. Assume that $\forall \beta<\alpha\left[\beta \in X_{d} \rightarrow(4) \wedge(5)\right]$ and that $G_{\alpha}(x) \leq d$. Hence $\alpha_{x} \in X_{d}$ and thus $\alpha_{x} \Rightarrow_{0} 0$. For $y<x$ it follows that $\alpha_{x} \Rightarrow_{0} \alpha_{y}$. Thus by the inductive assumption applied successively to $\alpha_{x},\left(\alpha_{x}\right)_{0},\left(\left(\alpha_{x}\right)_{0}\right)_{0}, \ldots, \alpha_{y}$ we infer that $G_{\alpha_{y}}(x)<G_{\alpha_{x}}(x)$, i.e. $G_{\alpha_{y}}(x)<G_{\alpha}(x)$ and (5) is proved.

Since by the inductive assumption $G_{\alpha_{y}}$ is increasing, also $G_{\alpha_{y}}(y)<$ $G_{\alpha_{x}}(x)$, i.e. $G_{\alpha}(y)<G_{\alpha}(x)$, which finishes the proof.

Proof of (6). Assume that $\forall x \in \operatorname{dm} F[G(x) \downarrow \rightarrow F(x) \leq G(x)]$ and fix $d$. It is enough to show that $\forall \alpha \forall x \geq 1\left[G_{\alpha}(x) \leq d \rightarrow F_{\alpha}(x) \leq G_{\alpha}(x)\right]$. We show this by induction on $\alpha \in X_{d}$.

If $\alpha \in \operatorname{Lim}$ and $x \geq 1, G_{\alpha}(x) \leq d$, then obviously by the inductive assumption $F_{\alpha}(x) \simeq F_{\alpha_{x}}(x) \leq G_{\alpha_{x}}(x) \simeq G_{\alpha}(x)$. Assume now that $\alpha=\beta+1$ and $G_{\alpha}(x) \leq d$. Then $G_{\beta}(F(x)) \leq d$ by $(4)$, because $G_{\beta}(F(x)) \leq$ $G_{\beta}(G(x)) \simeq G_{\alpha}(x) \leq d$. It follows by the inductive assumption that $F_{\beta}(F(x)) \leq G_{\beta}(F(x))$ and finally $F_{\alpha}(x) \simeq F_{\beta}(F(x)) \leq G_{\alpha}(x)$.

Proof of (8). We argue by induction on $\beta$, which reduces to finite induction. Since by the definition $G_{\beta+1} \simeq G_{\beta} \circ G_{1}$, the successor step is immediate. The limit step follows from (2) of Definition 1.5.

This finishes the proof.

We need some more facts about hierarchies. Assume that PA $\vdash$ " $G$ is a function". We define in PA a hierarchy $\bar{G}_{\alpha}$ of functions growing a little faster than $G_{\alpha}$, as follows: 
(1) $\bar{G}_{0}=\mathrm{id}$,

(2) $\quad \bar{G}_{\alpha}(x) \simeq \bar{G}_{\alpha_{G(x)}}(G(x))$,

(3) $\bar{G}_{\alpha}(x) \downarrow \Leftrightarrow$ we can compute $\bar{G}_{\alpha}(x)$ in a finite number of steps.

3.2. Lemma (PA).

(1) If $G$ is increasing and $\forall x \in \operatorname{dm} G(0<x<G(x))$, then for all $x$

$$
G_{\alpha}(x) \leq \bar{G}_{\alpha}(x) \leq G_{\alpha+2}(x) .
$$

(2) $\quad$ Let $\overline{T H}\left(\varepsilon_{\alpha}\right)=\mathrm{PA}+\left\{\forall x \exists y\left(\overline{H^{n}}\right)_{\varepsilon_{\alpha}}(x)=y: n \in \omega\right\}$. Then $\overline{T H}\left(\varepsilon_{\alpha}\right) \equiv$ $T H\left(\varepsilon_{\alpha}\right)$.

(3) For all $n, m$ satisfying $n \leq m$ we have $T H\left(\left(\varepsilon_{\alpha}\right)_{n}\right) \subseteq T H\left(\left(\varepsilon_{\alpha}\right)_{m}\right)$.

Proof. (1) The first inequality follows by induction on $\alpha$ from 3.1(4), (5); we need only observe that the number of $\alpha$ with $\exists x \bar{G}_{\alpha}(x) \leq d$ is finite.

Now we prove the second inequality by induction on $\alpha$. Assume that $\alpha=\beta+1$ and $\bar{G}_{\beta}(x) \leq G_{\beta+2}(x)$ for all $x$. Then $\bar{G}_{\alpha}(x)=\bar{G}_{\beta}(G(x)) \leq$ $G_{\beta+2}(G(x)) \simeq G_{\alpha+2}$. For $\alpha \in \operatorname{Lim}$ assume that the inequality holds for numbers $<\alpha$. Then

$$
\bar{G}_{\alpha}(x)=\bar{G}_{\alpha_{G(x)}}(G(x)) \leq G_{\alpha_{G(x)+2}}(G(x)) \simeq G_{\alpha_{G(x)+1}}\left(G^{2}(x)\right) .
$$

Since $\alpha_{G^{2}(x)} \Rightarrow_{0} \alpha_{G(x)}+1$, it follows by 3.1(5) that

$$
G_{\alpha_{G(x)+1}}\left(G^{2}(x)\right) \leq G_{\alpha_{G^{2}(x)}}\left(G^{2}(x)\right) \simeq G_{\alpha}\left(G^{2}(x)\right) \simeq G_{\alpha+2} .
$$

(2) is an immediate consequence of (1) and 3.1(8).

(3) Let $n<m$ and let $k$ be arbitrary. We work in the system $T H\left(\left(\varepsilon_{\alpha}\right)_{m}\right)$. Obviously there exists a $d$ such that $d=H_{\left(\varepsilon_{\alpha}\right)_{m}}^{k}(1)$. From this we infer by $3.1(3)$ that $\left(\varepsilon_{\alpha}\right)_{m} \Rightarrow_{0} 0$. Hence by $3.1(1),\left(\varepsilon_{\alpha}\right)_{m} \Rightarrow_{0}\left(\varepsilon_{\alpha}\right)_{n}$. It follows by $3.1(5)$ that $\forall x>0 H_{\left(\varepsilon_{\alpha}\right)_{n}}^{k}(x)<H_{\left(\varepsilon_{\alpha}\right)_{m}}^{k}(x)$ and hence in particular $\forall x>0$ $H_{\left(\varepsilon_{\alpha}\right)_{n}}^{k}(x) \downarrow$.

The proof of Lemma 1.13 is based on the logical properties of finite sets of diagonally indiscernible elements. We now recall the relevant definitions and lemmas from [8].

Let $\theta^{*}\left(\bar{x}, y_{1}, \ldots, y_{n}\right)$ denote the $\Delta_{0}$-formula of class $\Delta_{0}$ obtained from $\theta(\bar{x}) \in L_{\mathrm{PA}}$ by bounding the quantifiers of the deepest uniform blocks by $y_{n}$, those of the subsequent blocks by $y_{n-1}$ and so on; the last block will be bounded by $y_{1}$. The number $n$ is called the arithmetical range of the formula $\theta$ and is denoted by a.r. $(\theta)$.

3.3. Definition (PA). We say that a finite set $A$ is an a-skeleton iff

(1) $|A|>\min A \geq a$,

(2) $A$ is a set of diagonally indiscernible elements for all $\theta^{*}$ such that $\theta$ is a generalization of an $\eta<a$. 
Recall that $A$ is a set of diagonally indiscernible elements for $\theta^{*}(x)$ (where a.r. $(\theta)=n$ ) iff

$$
\begin{aligned}
\forall a \in A \forall \bar{b}<a \forall \bar{c}, \bar{d}\{\bar{c}, \bar{d} \in[A \backslash & {[0, a]]^{n} } \\
& \left.\rightarrow\left[\operatorname{Tr}_{\Delta_{0}}\left(\theta^{*}(\bar{b}, \bar{c})\right) \Leftrightarrow \operatorname{Tr}_{\Delta_{0}}\left(\theta^{*}(\bar{b}, \bar{d})\right)\right]\right\} .
\end{aligned}
$$

In the case when the sequence $\bar{x}$ is empty the quantifier $\forall \bar{b}<a$ drops out, we also drop $\forall a \in A$ and put $a=-1$ and by the diagonal indiscernibility of elements of $A$ we mean the usual indiscernibility of elements of $A$ for $\operatorname{Tr}_{\Delta_{0}}\left(\theta^{*}(\bar{y})\right)$.

Assume that $A$ is an $a$-skeleton, $\theta<a$, a.r. $(\theta)=n$ and that $A=$ $\left\{a_{0}, \ldots, a_{k}\right\}_{<}$. In that case we write $A \vDash \theta[\bar{x}]$ instead of $\operatorname{Tr}_{\Delta_{0}}\left(\theta^{*}\left(\bar{x}, a_{k-n+1}\right.\right.$, $\left.\left.\ldots, a_{k}\right)\right)$.

We assume additionally that formulas are coded in such a way that a.r. $(\theta) \leq \theta$ for all $\theta$, and subformulas have smaller codes.

3.4. OBSERVATION. Under the same assumption as above the following equivalences are true:

(1) $A \vDash \theta[\bar{x}]$ iff $\operatorname{Tr}_{\Delta_{0}}\left(\theta^{*}\left(\bar{x}, a_{1}, \ldots, a_{n}\right)\right)$ for $\bar{x}<a_{0}$.

(2) $A \vDash \theta$ iff $\operatorname{Tr}_{\Delta_{0}}\left(\theta^{*}\left(a_{0}, \ldots, a_{n-1}\right)\right)$ if the sequence $\bar{x}$ is empty.

(3) $A \vDash \forall \bar{x} \theta(\bar{x})$ iff $\forall \bar{x}<a_{0} A \vDash \theta[\bar{x}]$.

(4) $\forall \bar{x}_{2}<a_{0}\left[\left(A \vDash \forall \bar{x}_{1} \theta\left(\bar{x}_{1}, \bar{x}_{2}\right]\right)\right.$ iff $\left.\forall \bar{x}_{1}<a_{1} A \vDash \theta\left[\bar{x}_{1}, \bar{x}_{2}\right]\right]$.

(5) $A \vDash\left(\theta_{1} \vee \theta_{2}\right)[\bar{x}]$ iff $\left(A \vDash \theta_{1}[\bar{x}]\right) \vee\left(A \vDash \theta_{2}[\bar{x}]\right) \quad$ if $\theta_{1} \vee \theta_{2}<a$.

(6) $A \vDash \neg \theta[\bar{x}]$ iff $\neg(A \vDash \theta[\bar{x}]) \quad$ if $\neg \theta<a$.

In particular,

$$
A \vDash \forall \bar{x} \theta(\bar{x}) \text { iff } \forall \bar{x}<a_{0} A \vDash \theta[\bar{x}] \text { iff } \forall \bar{x}<a_{1} A \vDash \theta[\bar{x}] .
$$

Observe, moreover, that if the formula

$$
\operatorname{Ind}(\theta):=\forall \bar{y}[\eta(0, \bar{y}) \wedge \forall x(\eta(x, \bar{y}) \rightarrow \eta(x+1, \bar{y})) \rightarrow \forall x \eta(x, \bar{y})]
$$

is less than $a$, then $A \vDash \operatorname{Ind}(\theta)$.

Indeed, assume that $\bar{y}<a_{0}, A \vDash \eta[0, \bar{y}]$ and $A \vDash \forall x(\eta(x, \bar{y}] \rightarrow \eta(x+1, \bar{y}])$. Hence by $3.4(4-6), \forall x<a_{1}(A \vDash \eta[x, \bar{y}] \rightarrow A \vDash \eta[x+1, \bar{y}])$. Thus $\forall x<a_{1}$ $A \vDash \eta[x, \bar{y}]$. In consequence, $A \vDash \forall x \eta[x, \bar{y}]$ and finally by $3.4(3)$ we infer that $A \vDash \operatorname{Ind}(\theta)$.

Assume that $\Phi$ is a set of sentences of $L_{\mathrm{PA}}$ and let $A$ be an $a$-skeleton. Then we write $A \vDash_{a} \Phi$ iff $\forall \theta \in \Phi(\theta<a \rightarrow A \vDash \theta)$. Hence we can write

(7) For every $a$-skeleton $A, A \vDash_{a} \mathrm{Ax}(\mathrm{PA})$.

3.5. Definition (PA). We write $\Phi \vdash_{a} \theta$ if the formula $\theta$ has a proof within $\Phi$ such that all the formulas in that proof are less than $a$ (the proof has width less than $a$ ). 
We assume that the relation $\Phi \vdash \theta$ is defined on the base of the Hilbert type of proof with the rules MP, GN and the following axioms concerning quantifiers (cf. Lyndon's Notes on Logic):

$$
\forall x(\eta \rightarrow \theta) \rightarrow(\eta \rightarrow \forall x \theta), \quad x \notin \mathrm{fv}(\eta),
$$

and $\forall x \eta \Rightarrow \eta(t)$ where $t$ ranges only over simple terms of the form $0,1, x$, $x+y, x \cdot y$ (the language is $\left.L_{\mathrm{PA}}\right)$.

Assume now that PA $\vdash$ " $G$ is total".

3.6. Definition (PA). We say that a finite set $A$ is $G$-scattered iff $\forall a, b \in A[a<b \rightarrow G(a)<b]$. The sets which are $H^{n}$-scattered will also be called $\Sigma_{n}$-scattered.

3.7. Soundness Lemma (PA). Assume that $A$ is an $(x+1)^{2}$-scattered a-skeleton. Then for every set $\Phi$ of sentences and for each formula $\theta(\bar{x})$ the following implication is true:

$$
\Phi \vdash_{a} \theta \rightarrow\left(A \vDash_{a} \Phi \rightarrow A \vDash \forall \bar{x} \theta\right) .
$$

Sketch of proof. It is obvious that the set $\left\{\theta: A \vDash_{a} \forall \bar{x} \theta\right\}$ is closed under MP and GN. Hence it is enough to check that all logical axioms having codes less than $a$ are satisfied in $A$.

We will only check that

$$
A \vDash \forall \bar{x}[\forall y(\eta \rightarrow \theta) \rightarrow(\eta \rightarrow \forall y \theta)]
$$

under the assumption that the formula is less than $a$ and $y \notin \mathrm{fv}(\eta)$. Let $A=\left\{a_{0}, \ldots, a_{k}\right\}_{<}$. By 3.4(3) it is enough to show that

$$
\forall \bar{x}<a_{0} A \vDash[\forall y(\eta \rightarrow \theta) \rightarrow(\eta \rightarrow \forall y \theta)][\bar{x}] .
$$

Fix $\bar{x}<a_{0}$ and assume that $A \vDash \forall y(\eta \rightarrow \theta)[\bar{x}]$, i.e. in view of 3.4(4) that $\forall y<a_{1} A \vDash(\eta \rightarrow \theta)[\bar{x}, y]$. Hence

$$
\forall y<a_{1}(A \vDash \eta[\bar{x}] \rightarrow A \vDash \theta[\bar{x}, y]),
$$

i.e.

$$
A \vDash \eta[\bar{x}] \rightarrow \forall y<a_{1} A \vDash \theta[\bar{x}, y],
$$

which is equivalent to

$$
A \vDash \eta[\bar{x}] \rightarrow A \vDash \forall y \theta[\bar{x}, y],
$$

and this in view of 3.4(3) finishes our proof.

Finally, let us cite one more lemma from [8], Lemma II.2.4. Let $\varphi$ ᄀ denote the formula in normal form which is obtained in the natural way from $\neg \varphi$, when $\varphi$ is in normal form. 
3.8. Absoluteness Lemma (PA). Assume that a set $A$ is $\Sigma_{n}$-scattered, $|A|>\min A, \varphi$ is of class $\Sigma_{n}\left(\right.$ or $\left.\Pi_{n}\right)$ and $\varphi, \varphi \neg$ are less than $\min A$. Then for every $\bar{b}$, if above $\bar{b}$ in $A$ there are at least a.r. $(\varphi)+1$ elements, we have

$$
(A \vDash \varphi[\bar{b}]) \Leftrightarrow \operatorname{Tr}_{\Sigma_{n} \cup \Pi_{n}}(\varphi, \bar{b}) .
$$

4. Proof of the main lemma. Here we prove our main Lemma 1.13:

PA proves that for every $a<\lambda$ and for each $m$

(i) $T H\left(\varepsilon_{\alpha}\right) \vdash R\left(\bigcup_{n \in \omega} T H\left(\left(\varepsilon_{\underline{\alpha}}\right)_{n} ; \Sigma_{m}\right)\right) \quad$ if $\alpha$ is limit,

(ii) $T H\left(\varepsilon_{\alpha}\right) \vdash R\left(H\left(\varepsilon_{\beta}\right) ; \Sigma_{m}\right) \quad$ if $\alpha=\beta+1$.

Proof. It is enough to show in PA that for every fixed $\alpha<\lambda$ and fixed $\varphi(x) \in \Sigma_{m}$

(1) $\quad T H\left(\varepsilon_{\alpha}\right) \vdash \forall x\left[\left(\bigcup_{n \in \omega} T H\left(\left(\varepsilon_{\underline{\alpha}}\right)_{n}\right) \vdash\ulcorner\varphi\urcorner(\underline{x})\right) \rightarrow \varphi(x)\right] \quad$ if $\alpha \in \operatorname{Lim}$,

(2) $\quad T H\left(\varepsilon_{\alpha}\right) \vdash \forall x\left[\left(T H\left(\varepsilon_{\underline{\beta}}\right) \vdash\ulcorner\varphi\urcorner(\underline{x})\right) \rightarrow \varphi(x)\right] \quad$ if $\alpha=\beta+1$.

To do this we work now in the theory $T H\left(\varepsilon_{\alpha}\right)$. Take an arbitrary $x$ and assume that

$$
\begin{aligned}
\bigcup_{n \in \omega} T H\left(\left(\varepsilon_{\underline{\alpha}}\right)_{n}\right) \vdash\ulcorner\varphi\urcorner(\underline{x}) \quad \text { if } \alpha \in \operatorname{Lim}, \\
T H\left(\varepsilon_{\beta}\right) \vdash\ulcorner\varphi\urcorner(\underline{x}) \quad \text { if } \underline{\alpha}=\beta+1 .
\end{aligned}
$$

Hence in the limit case by $3.2(3)$ there exists an $l$ such that

$$
T H\left(\left(\varepsilon_{\underline{\alpha}}\right)_{l}\right) \vdash\ulcorner\varphi\urcorner(\underline{x}) .
$$

Set in this case $\varepsilon_{\beta}=\left(\varepsilon_{\underline{\alpha}}\right)_{l}$. By Lemma 3.2(2) we have $\overline{T H}\left(\varepsilon_{\beta}\right) \vdash\ulcorner\varphi\urcorner(\underline{x})$; obviously this is true in both cases. And there exists a number $n$ such that

(3) $\overline{T H}\left(\varepsilon_{\beta}\right) \vdash_{n}\ulcorner\varphi\urcorner(\underline{x})$.

Our task is to show

(4) There is a $\Sigma_{m}$-scattered $n$-skeleton $B$ such that $B \vDash_{n} \overline{T H}\left(\varepsilon_{\beta}\right)$ and $\min B>\ulcorner\varphi\urcorner \neg$.

Then by the Soundness Lemma 3.7 we have

$$
B \vDash\ulcorner\varphi\urcorner(x)
$$

and hence by the Absoluteness Lemma 3.8 the formula $\varphi(x)$ is true, which proves the corresponding statements in (1) and (2).

We have replaced $T H\left(\varepsilon_{\beta}\right)$ by $\overline{T H}\left(\varepsilon_{\beta}\right)$ for technical reasons. In the sequel (in the proof of Lemma 4.1) we essentially use the fact that the number of reduction steps in the computation of $y=(\bar{H})_{\varepsilon_{\beta}}(x)$ is $\leq y$. 
The first step in the proof of (4) is the following :

(5) Claim. For every a there exists an $\varepsilon_{\beta+1}$-large and $\Sigma_{m}$-scattered set A such that $\min A \geq a$.

Take an arbitrary number $a_{0}>a, l+1$. We define $A=\left\{\left(H^{m}\right)^{i}\left(a_{0}\right):\left(H^{m}\right)^{i}\left(a_{0}\right) \leq\left(H^{m}\right)_{\varepsilon_{\alpha}}\left(a_{0}\right) \wedge i\right.$ is a natural number $\}$.

Then $a_{0}=\min A$. Observe that $S^{A}(x)=H^{m}(x)$ for $x \in A \backslash\{\max A\}$, whence $\left(S^{A}\right)_{\varepsilon_{\alpha}}\left(a_{0}\right) \downarrow$ and so $A$ is $\varepsilon_{\alpha}$-large. Moreover, $A$ is $\Sigma_{m}$-scattered.

Now assume temporarily that $\alpha$ is limit. Since $l+1 \leq a_{0}$, it follows by $3.1(7)$ that $A$ is $\left(\varepsilon_{\alpha}\right)_{l+1}$-large. By $3.1(3)$ we infer that $\left(\varepsilon_{\alpha}\right)_{l+1} \Rightarrow_{0} 0$. Hence by $3.1(2)$

$$
\left(\varepsilon_{\alpha}\right)_{l+1} \Rightarrow_{0} \varepsilon_{\alpha_{l}^{\prime}+1}, \quad \text { i.e. } \quad\left(\varepsilon_{\alpha}\right)_{l+1} \Rightarrow_{0} \varepsilon_{\beta+1},
$$

because $\left(\varepsilon_{\alpha}\right)_{l}=\varepsilon_{\alpha_{l}^{\prime}}=\varepsilon_{\beta}$. Therefore $A$ is $\varepsilon_{\beta+1}$-large; the same is true in the nonlimit case.

Further, we need a refinement of Lemma II.3.4(ii) of [8] which says that PA proves: If $A$ is $\omega_{a}^{\varepsilon_{\beta}+1}$-large and $\min A \geq a \geq 3$, then there exists an $\varepsilon_{\beta}+1$-large $a$-skeleton $B \subseteq A$.

In fact, the same proof shows that there exists an $\omega^{\varepsilon_{\beta}+1}$-large $a$-skeleton $B \subseteq A$, which moreover is $2_{3}^{x}$-scattered.

By $1.5(3),\left(\omega^{\varepsilon_{\beta}+1}\right)_{1}=\varepsilon_{\beta} \cdot 2+1$. Moreover, by 3.1(3), $\varepsilon_{\beta}+1 \Rightarrow_{0} 0$ and hence $\varepsilon_{\beta}+1 \Rightarrow_{0} \varepsilon_{0} \Rightarrow_{0} \omega \Rightarrow_{2} 4$. Hence summing up $\omega^{\varepsilon_{\beta}+1} \Rightarrow_{\leq 2} \varepsilon_{\beta}+4$ and by $3.1(7)$ every $\omega^{\varepsilon_{\beta}+1}$-large set $B$ with $\min B \geq 2$ is also $\varepsilon_{\beta}+4$-large. Therefore we have the following refinement:

4.1. Lemma (PA). If $A$ is $\omega_{a}^{\varepsilon_{\beta}+1}$-large with $\min A \geq a \geq 3$, then there exists an $\varepsilon_{\beta}+4$-large and $2_{3}^{x}$-scattered a-skeleton $B \subseteq A$. Since $\left(\varepsilon_{\beta+1}\right)_{a-1}=$ $\omega_{a}^{\varepsilon_{\beta}+1}$, the assumption " $A$ is $\omega_{a}^{\varepsilon_{\beta}+1}$-large" can be strengthened to " $A$ is $\varepsilon_{\beta+1}$-large".

Now we make the following convention: we call $A \Sigma_{0}$-scattered iff $A$ is $2_{3}^{x}$-scattered. It follows by (5) and 4.1 that

(6) For every $a$ there is a $\Sigma_{m}$-scattered and $\varepsilon_{\beta}+4$-large $a$-skeleton $B$.

Obviously this is valid for all standard $m$.

To finish the proof of (4) it is enough by (6) to show the following:

4.2. LEMmA. Assume that the formula $P$ (which defines a system of sequences) is of class $\Sigma_{m+1}$. There exists a number $c$ such that PA proves that for every $\Sigma_{m}$-scattered set $B$ the following implication is valid:

" $B$ is an $\varepsilon_{\beta}+4$-large $n^{c}$-skeleton" $\rightarrow B \vDash_{n} \overline{T H}\left(\varepsilon_{\beta}\right)$.

We have formulated the above statement as a separate lemma, mainly because it will also be useful in the next section. 
Proof. We choose an arbitrary number $c$ such that PA proves (1) and (2) below:

(1) For every $k$ and every $n$

$$
\left\ulcorner\forall x \exists y H^{k}(x)=y\right\urcorner<n \rightarrow \mathrm{PA} \vdash_{n^{c}} \forall x \exists y H^{k}(x)=y,
$$

(2) $\quad \mathrm{PA} \vdash_{\underline{c}} \forall \alpha \forall x \exists \gamma(P(\alpha, x)=\gamma) \wedge \exists \gamma\left(\varepsilon_{\underline{\beta}}=\gamma\right)$.

Now we work in PA. Let the sentence $\forall x \exists y\left(\overline{H^{k}}\right)_{\varepsilon_{\underline{\beta}}}(x)=y$ be an axiom of the theory $\overline{T H}\left(\varepsilon_{\beta}\right)$ with code less than $n$ and let $B$ satisfy the assumptions of the lemma. The lemma will be proved if we show that

(3) the formula $\left(\forall x \exists y\left(\overline{H^{k}}\right)_{\varepsilon_{\beta}}(x)=y\right)^{*}$ is satisfied by some increasing sequence of elements of $\overline{B^{-}}$.

By 3.4(7), $B \vDash_{n^{c}} \operatorname{Ax}(\mathrm{PA})$. Since $B$ is $(x+1)^{2}$-scattered, it follows by the Soundness Lemma 3.7 that $B \vDash \forall x \exists y H^{k}(x)=y$. We define a partial function by

$$
F(x)=\min _{y}\left\{B \vDash H^{k}(x)=y\right\} .
$$

Let $b_{0}, b_{1}, \ldots$ denote the elements of $B$ in increasing order. We denote the successor function $S^{B}$ in $B$ simply by $S$. Further, $\left(S^{B}\right)_{\gamma}\left(b_{0}\right)$ will be denoted simply by $b_{\gamma}$.

Our auxiliary aim is to find a reasonable upper bound for $\bar{F}_{\varepsilon_{\beta}}\left(b_{0}\right)$. In the subsequent estimations the following is very useful:

(4) Claim. $\forall \alpha\left[S_{\omega+\alpha}\left(b_{i+1}\right) \downarrow \rightarrow S_{\omega}\left(S_{\alpha}\left(b_{i}\right)\right) \leq S_{\omega+\alpha}\left(b_{i+1}\right)\right]$.

The initial step for $\alpha=0$ is obvious. The nonlimit step is very easy. Let $\alpha \in \operatorname{Lim}$. If $\alpha \neq \omega^{2}$, then it is easy to check that $(\omega+\alpha)_{b_{i+1}}=\omega+\alpha_{b_{i+1}}$. However, for $\alpha=\omega^{2}$, we have

$$
(\omega+\alpha)_{b_{i+1}}=\left(\omega^{2}\right)_{b_{i+1}}=\omega\left(b_{i+1}+1\right)+1 \Rightarrow_{0} \omega+\omega\left(b_{i}+1\right)+1=\omega+\alpha_{b_{i}} .
$$

Therefore

$$
\begin{aligned}
S_{\omega+\alpha}\left(b_{i+1}\right) & =S_{(\omega+\alpha)_{b_{i+1}}}\left(b_{i+1}\right) \\
& \geq S_{\omega+\alpha_{b_{i}}}\left(b_{i+1}\right) \geq S_{\omega}\left(S_{\alpha_{b_{i}}}\left(b_{i}\right)\right)=S_{\omega}\left(S_{\alpha}\left(b_{i}\right)\right) .
\end{aligned}
$$

By (4), $S_{\omega}\left(b_{\varepsilon_{\beta}+2}\right) \leq b_{\varepsilon_{\beta}+3}$. Hence there exist at least a.r. $\left(H^{k}\right)$ elements above $b_{\varepsilon_{\beta}+2}$ in $B$, because a.r. $\left(H^{k}\right)<b_{0}$. It follows by the definition of the relation of satisfaction that

$$
\forall b \in B \cap\left[0, b_{\varepsilon_{\beta}+2}\right] \forall x \leq b \exists y \leq S(b) B \vDash H^{k}(x)=y .
$$

Hence

$$
\forall b \in B \cap\left[0, b_{\varepsilon_{\beta}+2}\right] \forall x \leq b F(x) \leq S(b) .
$$


By 3.2(1), $\bar{F}_{\varepsilon_{\beta}}\left(b_{0}\right) \leq F_{\varepsilon_{\beta}+2}\left(b_{0}\right)$. By $(5)$ and 3.1(6), $F_{\varepsilon_{\beta}+2}\left(b_{0}\right) \leq$ $S_{\varepsilon_{\beta}+2}(b)$. Therefore finally

$$
\bar{F}_{\varepsilon_{\beta}}\left(b_{0}\right) \leq b_{\varepsilon_{\beta}+2} .
$$

Now we show that the formula in (3) is satisfied by the sequence $b_{0}, b_{\varepsilon_{\beta}+3}$, $S\left(b_{\varepsilon_{\beta}+3}\right), \ldots, S^{r}\left(b_{\varepsilon_{\beta}+3}\right)$ for some $r<b_{0}$.

We will need an exact form of the formula $\forall x \exists y\left(\overline{H^{k}}\right)_{\varepsilon_{\underline{\beta}}}(x)=y$. The general scheme of this formula is $\forall x \exists y \exists w \varphi(x, y, w)$, where $\bar{w}$ is the sequence which witnesses $\left(\overline{H^{k}}\right)_{\varepsilon_{\beta}}(x)=y$. The formula $\varphi(x, y, w)$ can be written explicitly as follows:

$$
\begin{aligned}
\exists s\{\operatorname{Seq}(w) & \wedge \operatorname{lh}(w)=s \wedge(w)_{0}=\left\langle x, \varepsilon_{\underline{\beta}}\right\rangle \wedge(w)_{s}=\langle y, 0\rangle \\
& \wedge \forall i, t, u \forall \gamma, \delta\left[i<s \wedge\left(w_{i}\right)_{0}=t \wedge\left(w_{i+1}\right)_{0}=u \wedge\left(w_{i}\right)_{1}=\gamma\right. \\
& \left.\left.\wedge\left(w_{i+1}\right)_{1}=\delta \rightarrow \gamma \neq 0 \wedge u=H^{k}(t) \wedge \delta=P(\gamma, u)\right]\right\} .
\end{aligned}
$$

By (4) there are at least $b_{\varepsilon_{\beta}+3}$ elements above $b_{\varepsilon_{\beta}+3}$ in $B$. Hence to finish our proof it is enough to show

(7) Claim. For each $x<b_{0}$ there exist $y<b_{\varepsilon_{\beta}+3}$ and $w<b_{\varepsilon_{\beta}+3}$ such that $B \vDash \varphi(x, y, w)$.

Fix $x<b_{0}$ and let $y=\bar{F}_{\varepsilon_{\beta}}(x)$. Hence by $(6), y \leq b_{\varepsilon_{\beta}+2}$. Let $w$ be the sequence which witnesses $y=\bar{F}_{\varepsilon_{\beta}}(x)$. Therefore

$$
w=\left\langle\left\langle t_{0}, \beta^{0}\right\rangle, \ldots,\left\langle t_{s}, \beta^{s}\right\rangle\right\rangle,
$$

where $t_{0}=x, \beta^{0}=\varepsilon_{\beta}, t_{s}=y, \beta^{s}=0$ and for all $i<s$

$$
t_{i+1}=F\left(t_{i}\right), \quad \beta^{i+1}=\left(\beta^{i}\right)_{t_{i+1}} \wedge \beta^{i} \neq 0 .
$$

The sequence $t_{i}: i \leq s$ is increasing and hence $s \leq y \leq b_{\varepsilon_{\beta}+2}$. Moreover, obviously $t_{i} \leq y$ for all $i \leq s$.

Now we find an upper bound for $\left\ulcorner\beta^{i}\right\urcorner$ and finally for $w$. We show inductively that

$$
\forall i \leq s\left\ulcorner\beta^{i}\right\urcorner<S^{i}\left(b_{\varepsilon_{\beta}+2}\right) .
$$

(We write $\left\ulcorner\beta^{i}\right\urcorner$ whenever we treat $\beta^{i}$ simply as a natural number.)

By the assumption $A$ is $\Sigma_{m}$-scattered and $P \in \Sigma_{m+1}$. In particular, $P(\alpha, x)=\gamma$ is of the form $\exists z P^{\prime}(\alpha, x, \gamma, z)$ for some $P^{\prime} \in \Pi_{m}$.

From (2), by the Soundness Lemma 3.7 it follows that there exists a $\gamma$ such that $\ulcorner\gamma\urcorner<b_{0}$ and $B \vDash \varepsilon_{\beta}=\gamma$. Since $\Sigma_{1}$-formulas are upward absolute we obtain $\gamma=\varepsilon_{\beta}$. Hence for $i=0$, we have $\left\ulcorner\beta^{0}\right\urcorner=\left\ulcorner\varepsilon_{\beta}\right\urcorner \leq b_{0}$. Assume now inductively that $\left\ulcorner\beta^{i}\right\urcorner<S^{i}\left(b_{\varepsilon_{\beta}+2}\right)$ for $i<s$. From (2), by the Soundness Lemma 3.7 , it also follows that

$$
B \vDash \forall \alpha \forall x \exists \gamma \exists z P^{\prime}(\alpha, x, \gamma, z) .
$$


Hence by Observation 3.4(4)

$$
\forall\ulcorner\alpha\urcorner, x<S^{i}\left(b_{\varepsilon_{\beta}+2}\right) \exists\ulcorner\gamma\urcorner, z<S^{i+1}\left(b_{\varepsilon_{\beta}+2}\right) B \vDash P^{\prime}(\alpha, x, \gamma, z) .
$$

Then we put $\alpha=\beta^{i}, x=t^{i+1}$. By the Absoluteness Lemma 3.8, $(B \vDash$ $\left.P^{\prime}(\alpha, x, \gamma, z)\right) \Leftrightarrow P^{\prime}(\alpha, x, \gamma, z)$ for all $\ulcorner\gamma\urcorner, z<S^{i+1}\left(b_{\varepsilon_{\beta}+2}\right)$. It follows that $\left\ulcorner\beta^{i+1}\right\urcorner=\left\ulcorner\left(\beta^{i}\right)_{t_{i+1}}\right\urcorner<S^{i+1}\left(b_{\varepsilon_{\beta}+2}\right)$, which finishes the proof of (8).

Moreover, we have proved that

$$
\forall i<s B \vDash P\left(\beta^{i}, t_{i+1}\right)=\beta^{i+1} .
$$

By (8) we see that all terms of the sequence $w$ are less than $\left\langle S^{s}\left(b_{\varepsilon_{\beta}+2}\right)\right.$, $\left.b_{\varepsilon_{\beta}+2}\right\rangle$ and that $s \leq b_{\varepsilon_{\beta}+2}$. Thus $w<S\left(S^{b_{\varepsilon_{\beta}+2}}\left(b_{\varepsilon_{\beta}+2}\right)\right)$, because $B$ is $2_{3}^{x}$ scattered. Finally, by $(4), w<S_{\omega}\left(b_{\varepsilon_{\beta}+2}\right) \leq b_{\varepsilon_{\beta}+3}$.

Observe additionally that

$$
B \vDash\left\langle x, \varepsilon_{\beta}\right\rangle=\left\langle x, \varepsilon_{\beta}\right\rangle=(w)_{0} \quad \text { and } \quad B \vDash \operatorname{Seq}(w) \wedge \operatorname{lh}(w)=s .
$$

The last statement is valid because $B$ is (at least) $2_{3}^{x}$-scattered and e.g. $\operatorname{Seq}(w)$ can be easily written as a $\Delta_{0}\left(2^{x}\right)$-formula bounded by $2_{3}^{w}$.

Summing up (8)-(10) we have proved that

$$
\begin{aligned}
\forall x<b_{0} & \exists y, w, s<b_{\varepsilon_{\beta}+3}\left[\left(B \vDash \operatorname{Seq}(w) \wedge \operatorname{lh}(w)=s \wedge(w)_{0}=\left\langle x, \varepsilon_{\underline{\beta}}\right\rangle\right.\right. \\
& \left.\wedge(w)_{s}=\langle y, 0\rangle\right) \wedge w=\left\langle\left\langle t_{0}, \beta^{0}\right\rangle, \ldots,\left\langle t_{s}, \beta^{s}\right\rangle\right\rangle \\
& \left.\wedge \forall i<s\left(B \vDash t_{i+1}=H^{k}\left(t_{i}\right) \wedge \beta^{i+1}=P\left(\beta^{i}, t_{i+1}\right) \wedge \beta^{i} \neq 0\right)\right] .
\end{aligned}
$$

By Observation 3.4(4) to finish the proof of Claim (7) it is enough to show that for all $i, t, u, \gamma, \delta<b_{\varepsilon_{\beta}+3}, B \vDash i<s \wedge\left(w_{i}\right)_{0}=t \wedge\left(w_{i}\right)_{1}=u$ implies $i<s \wedge\left(w_{i}\right)_{0}=t \wedge\left(w_{i}\right)_{1}=u$ etc. Since the formulas considered are of class $\Sigma_{1}$ they are obviously upward absolute. This finishes the proof of Lemma 4.2.

5. Remaining proofs. In this section we show the three yet unproved theorems announced in the introduction. We base on Theorem 1.12 proved in Section 2.

First we show Theorem 1.11: If the system of sequences $P$ is of class $\Sigma_{1}$ then

$$
I\left(\varepsilon_{\alpha}\right) \cap \Pi_{2} \equiv T H^{0}\left(<\varepsilon_{\alpha+1}\right) .
$$

The proof relies on the idea very similar to that in the proof of the main Lemma 1.13.

Proof of Theorem 1.11. Obviously $T H^{0}\left(<\varepsilon_{\alpha+1}\right) \subseteq I\left(\varepsilon_{\alpha}\right) \cap \Pi_{2}$, because $I\left(\varepsilon_{\alpha}\right) \equiv I\left(<\varepsilon_{\alpha+1}\right) \supseteq T H^{0}\left(<\varepsilon_{\alpha+1}\right)$ (see Gentzen [1]).

Now we prove the reverse inclusion. By Theorem 1.12 it is enough to show that $T H\left(\varepsilon_{\alpha}\right) \cap \Pi_{2} \subseteq T H^{0}\left(<\varepsilon_{\alpha+1}\right)$. Since $\overline{T H}\left(\varepsilon_{\alpha}\right) \equiv T H\left(\varepsilon_{\alpha}\right)$ it is enough to show that for every $\varphi(x, y) \in \Delta_{0}, \overline{T H}\left(\varepsilon_{\alpha}\right) \vdash \forall x \exists y \varphi(x, y)$ implies $T H^{0}\left(<\varepsilon_{\alpha+1}\right) \vdash \forall x \exists y \varphi(x, y)$. 
Fix $\varphi \in \Delta_{0}$ and assume that

$$
\overline{T H}\left(\varepsilon_{\alpha}\right) \vdash_{n} \forall x \exists y \varphi(x, y) .
$$

We will work in $T H^{0}\left(<\varepsilon_{\alpha+1}\right)$, just as we worked in $T H\left(\varepsilon_{\alpha}\right)$ in the proof of the main lemma.

Our aim now is to show

(1) For every $x_{0}$ there is an $\underline{n}$-skeleton $B$ such that $B \vDash_{\underline{n}} \overline{T H}\left(\varepsilon_{\alpha}\right)$ and $\min B>x_{0}$.

Fix $x_{0}$. Then by the Soundness Lemma 3.7 we have $B \vDash \forall x \exists y \varphi(x, y)$ and hence $\forall x<b_{0} \exists y<b_{1} \varphi(x, y)$ (where $b_{0}$ is the first and $b_{1}$ the second element of $B)$. Thus we have $\exists y \varphi\left(x_{0}, y\right)$ for all $x_{0}$ and theorem is proved.

Let $c$ be the standard constant from Lemma 4.2. Since $P \in \Sigma_{1}$ the proof of (1) reduces to finding a $2_{3}^{x}$-scattered and $\varepsilon_{\alpha}+4$-large $\underline{n}^{c}$-skeleton $B$. Let $\beta=\omega_{n^{c}}^{\varepsilon_{\alpha}+1}$. We define $A=\left[x_{0}+1, H_{\beta}\left(x_{0}+1\right)\right]$. It is easy to check that $\left(S^{A}\right)_{\beta}\left(x_{0}+1\right)=H_{\beta}\left(x_{0}+1\right)$ and hence $A$ is $\omega_{n^{c}}^{\varepsilon_{\alpha}+1}$-large. Hence our claim follows by Lemma 4.1.

Now we prove Theorems 1.14 and 1.15. Let us repeat their statements.

THEOREM 1.14. If the system of sequences $P$ is of class $\Sigma_{1}$ then

$$
\mathrm{PA} \vdash\left[\overline{P H}_{\alpha} \Leftrightarrow R\left(I\left(<\varepsilon_{\alpha}\right) ; \Sigma_{1}\right)\right] .
$$

THEOREM 1.15. If the system of sequences $P$ is of class $\Sigma_{1}$ then

$$
\mathrm{PA} \vdash\left[\forall x \exists y H_{\varepsilon_{\alpha}}(x)=y \Leftrightarrow R\left(I\left(<\varepsilon_{\alpha}\right) ; \Sigma_{1}\right)\right] .
$$

Since the general scheme of both proofs is the same, we prove the two theorems simultaneously.

Proof. We work in PA. For the proof of $1.15(\Leftarrow)$ we observe that

$$
\forall x \forall n\left[I\left(<\varepsilon_{\alpha}\right) \vdash \exists y H_{\left(\varepsilon_{\underline{\alpha}}\right)_{\underline{n}}}(\underline{x})=y\right] .
$$

Since $H_{\left(\varepsilon_{\alpha}\right)_{n}}(x)=y$ is of class $\Sigma_{1}$, by $R\left(I\left(<\varepsilon_{\alpha}\right) ; \Sigma_{1}\right)$ we see from (1) that $\forall n \forall x \exists y \bar{H}_{\left(\varepsilon_{\alpha}\right)_{n}}(x)=y$, which shows $1.15(\Leftarrow)$.

Now we prove $1.14(\Leftarrow)$. Observe that

$$
\forall \gamma \forall n[I(\gamma) \vdash \forall x \exists y([1, y] \rightarrow(H \text { is } \underline{\gamma} \text {-large } \wedge|H| \geq x+1) \underline{n})] .
$$

The proof is similar to the proof of

$$
\forall n[\mathrm{PA} \vdash \forall x \exists y([1, y] \rightarrow(|H| \geq \min H \wedge|H| \geq x+1) \underline{\underline{n}})]
$$

from [6], which is based on the infinite version of the Ramsey Theorem on partitions of $n$-tuples and on the König Lemma. We use the fact that

$$
I(\gamma) \vdash \text { " } A \text { is unbounded } \rightarrow \exists z A \cap[1, z] \text { is } \underline{\gamma} \text {-large" . }
$$


Hence

$$
\begin{gathered}
\forall x\left[I ( < \varepsilon _ { \alpha } ) \vdash \exists y \left([ 1 , y ] \rightarrow \left(H \text { is }\left(\varepsilon_{\underline{\alpha}}\right)_{\underline{x}}\right.\right.\right. \text {-large } \\
\wedge|H| \geq \underline{x}+1) \underline{\underline{x}})] \quad \text { if } \alpha \text { is limit, and } \\
\forall x, y\left[I ( < \varepsilon _ { \alpha } ) \vdash \exists z \left([ \underline { y } , z ] \rightarrow \left(H \text { is } \underline{\varepsilon}_{\underline{\beta}}\right.\right.\right. \text {-large } \\
\left.\left.\wedge|H| \geq \underline{x}+1)^{\frac{x}{x}}\right)\right] \quad \text { if } \alpha=\beta+1 .
\end{gathered}
$$

Since the formulas on the right hand side of $\vdash$ are of class $\Sigma_{1}$, by $R\left(I\left(<\varepsilon_{\alpha}\right) ; \Sigma_{1}\right)$ we see that $\overline{\mathrm{PH}}_{\alpha}$ is valid, which shows $1.14(\Leftarrow)$.

Now we show the opposite implications. To do this assume that

$$
\forall x \exists y H_{\varepsilon_{\alpha}}(x)=y \text {. }
$$

We use Theorem 1.12, which implies that

$$
\begin{array}{ll}
I\left(<\varepsilon_{\alpha}\right) \equiv \bigcup_{m} T H\left(\left(\varepsilon_{\alpha}\right)_{m}\right) & \text { if } \alpha \in \operatorname{Lim}, \\
I\left(<\varepsilon_{\alpha}\right) \equiv I\left(\varepsilon_{\beta}\right) \equiv T H\left(\varepsilon_{\beta}\right) & \text { if } \alpha=\beta+1 .
\end{array}
$$

Fix $\varphi(x) \in \Sigma_{1}$ and assume

$$
\begin{aligned}
\bigcup_{m} T H\left(\left(\varepsilon_{\alpha}\right)_{m}\right) \vdash\ulcorner\varphi\urcorner(\underline{x}) & \text { if } \alpha \in \operatorname{Lim}, \\
T H\left(\varepsilon_{\beta}\right) \vdash\ulcorner\varphi\urcorner(\underline{x}) & \text { if } \alpha=\beta+1 .
\end{aligned}
$$

If $\alpha \in \operatorname{Lim}$ then there exists an $l$ such that $T H\left(\left(\varepsilon_{\alpha}\right)_{l}\right) \vdash\ulcorner\varphi\urcorner(\underline{x})$. We know that there exists a $\beta<\alpha$ such that $\left(\varepsilon_{\alpha}\right)_{l}=\varepsilon_{\beta}$ and $\left(\varepsilon_{\alpha}\right)_{l+1} \Rightarrow_{0} \varepsilon_{\beta+1}$ (cf. proof of Lemma 1.13). For this $\beta$, TH( $\left.\varepsilon_{\beta}\right) \vdash\ulcorner\varphi\urcorner(\underline{x})$; the same is true for nonlimit $\alpha$, where $\alpha=\beta+1$. It follows that $\overline{T H}\left(\varepsilon_{\beta}\right) \vdash_{n}\ulcorner\varphi\urcorner(\underline{x})$ for some $n$.

Now our aim is to show the following:

(5) There is an $n$-skeleton $B$ such that $B \vDash_{n} \overline{T H}\left(\varepsilon_{\beta}\right)$.

Then $B \vDash \varphi(\underline{x})$ and obviously $\varphi(x)$ is valid, because $\varphi \in \Sigma_{1}$.

Let $c$ be the constant from Lemma 4.2. Now we consider our two implications separately.

Proof of $1.15(\Rightarrow)$. To prove (5) it is enough, by 4.2 , to show that there exists an $\varepsilon_{\beta}+4$-large and $2_{3}^{x}$-scattered $n^{c}$-skeleton $B$.

By (4) there exists an $\varepsilon_{\alpha}$-large set $A$ satisfying $n^{c}, l+1 \leq \min A$. We know that $\varepsilon_{\alpha} \Rightarrow \leq l+1 \varepsilon_{\beta+1}$. By 1.5(6), $\left(\varepsilon_{\beta+1}\right)_{n^{c}-1}=\omega_{n^{c}}^{\varepsilon_{\beta}+1}$. Hence $\varepsilon_{\alpha} \Rightarrow \leq a_{0}$ $\omega_{n^{c}}^{\varepsilon_{\beta}+1}$, where $a_{0}=\min A$, and by 3.1(7) it follows that $A$ is $\omega_{n^{c}}^{\varepsilon_{\beta}+1}$-large. Hence by 4.1 the required set $B$ exists. This finishes the proof of $1.15(\Rightarrow)$.

Proof of $1.14(\Rightarrow)$. Case 1: $\alpha$ is limit. Assume $\overline{\mathrm{PH}}_{\alpha}$. The first step is a repetition of the proof of the Paris-Harrington Theorem [6]. By careful inspection of the proof of the main theorem in [6], in particular of 
the constructions in the proofs of 2.11 and 2.15 (cf. [8], Section II.4), we can isolate the lemma given below. We formulate it using our terminology of $a$-skeletons. Let $f_{0}(x)=x+2$, and let $f_{n+1}(x)=f_{n}^{(x)}(2)$ (where $f_{n}^{(x)}$ is $f_{n}$ composed with itself $x$ times). One can check that $f_{2}(x) \geq 2^{x}$, and $f_{3}(x) \geq 2_{3}^{x}$ for $x \geq 6$. Let $h_{n}(x)=\max _{y}\left\{f_{n}(y) \leq x\right\}$.

5.1. Lemma (PA). Let $a$ and $M$ be given. There is a $P:[M]^{e} \rightarrow r$, where $e \geq a$ and $r$ depend only on a, such that if $X \subseteq[0, M]$ is relatively large $(|X| \geq \min X)$, has cardinality $>e$ and is homogeneous for $P$, then

(i) $X$ is $f_{3}$-scattered and $\min X \geq e$,

(ii) $h_{3}[X]$ minus last e elements is an a-skeleton.

Let $a=\max \left(n^{c}, l+1,6\right)$. Using $\overline{\mathrm{PH}}_{\alpha}$ we have, by 5.1, an $\left(\varepsilon_{\alpha}\right)_{e}$-large set $A$ such that the set $B=h_{3}[A]$ minus last $e$ elements is a $2_{3}^{x}$-scattered $a$-skeleton; obviously $B$ is an $n^{c}$-skeleton .

We put $A=\left\{a_{0}, \ldots, a_{k}\right\}_{<}$. Since $P$ is a $B^{+}$-system, we have $\left(\varepsilon_{\alpha}\right)_{e} \Rightarrow_{0}$ $\left(\varepsilon_{\alpha}\right)_{e-1}+1,\left(\varepsilon_{\alpha}\right)_{e-1} \Rightarrow_{0}\left(\varepsilon_{\alpha}\right)_{e-2}+1, \ldots,\left(\varepsilon_{\alpha}\right)_{l+2} \Rightarrow_{0}\left(\varepsilon_{\alpha}\right)_{l+1}+1$. It follows that $A-\left\{a_{0}\right\}$ is $\left(\varepsilon_{\alpha}\right)_{e-1}$-large, $A-\left\{a_{0}, a_{1}\right\}$ is $\left(\varepsilon_{\alpha}\right)_{e-2}$-large etc. Hence finally $A^{\prime}=A$ minus first $e-(l+1)$ elements is $\left(\varepsilon_{\alpha}\right)_{l+1}$-large. By the choice of $l$, $\left(\varepsilon_{\alpha}\right)_{l+1} \Rightarrow_{0} \varepsilon_{\beta+1}$. Thus $A^{\prime}$ is also $\varepsilon_{\beta+1}$-large.

Solovay and Ketonen [3] proved the following lemma:

(6) If $A^{\prime}=\left\{a_{0}^{\prime}, \ldots, a_{s}^{\prime}\right\}_{<}$is $\gamma$-large, then every $A^{\prime \prime}=\left\{a_{0}^{\prime \prime}, \ldots, a_{s}^{\prime \prime}\right\}_{<}$such that $\forall i \leq s \quad a_{i}^{\prime \prime} \leq a_{i}^{\prime}$ is also $\gamma$-large.

They proved this lemma for $\alpha \leq \varepsilon_{0}$, but it is provable in PA for an arbitrary recursive $\alpha$ endowed with a $B^{+}$-system of sequences.

Using this lemma we infer that $A^{\prime \prime}=A$ minus last $e-(l+1)$ elements is $\varepsilon_{\beta+1}$-large.

Now we easily check (see the beginning of the proof of 1.13) that $A^{\prime \prime}$ is $\varepsilon_{\beta}+5$-large. Hence the set $\bar{A}=A$ minus last $e$ elements is $\varepsilon_{\beta}+4$-large. Moreover, we have $B=h_{3}[\bar{A}]$ and $|B|=|\bar{A}|$.

By (6) we infer that $B$ is $\varepsilon_{\beta}+4$-large. And since $B$ is a $2_{3}^{x}$-scattered $n^{c}$-skeleton, we conclude by Lemma 4.2 that $B \vDash_{n} \bar{H}\left(\varepsilon_{\beta}\right)$, which finishes the proof of (5) in the limit case.

Case 2: $\alpha=\beta+1$. Now 5.1 is probably too weak to obtain the result. We need control over the magnitude of $e$. We use the refinement of 5.1 which was proved in [8] (see Lemma II.4.2). Roughly speaking, it says that for large $a$ there exists a $P:\left[2_{4}^{a}, M\right]^{a} \rightarrow 2$ with properties very similar to those in 5.1. It will cause no harm to simply assume that they are the same.

Recall that

$$
\overline{T H}\left(\varepsilon_{\beta}\right) \vdash_{n}\ulcorner\varphi\urcorner(\underline{x}) \quad \text { for some } n \text {. }
$$


Let $\overline{T H}{ }^{\prime}\left(\varepsilon_{\beta}\right)=\mathrm{PA} \cup\left\{\forall z \forall x \exists y\left(H^{k}\right)_{\left(\varepsilon_{\underline{\beta}}\right)_{z}}(x)=y: k\right.$ is a natural number $\}$. Hence also

$$
\overline{T H}^{\prime}\left(\varepsilon_{\beta}\right) \vdash_{n}\ulcorner\varphi\urcorner(\underline{x}) \quad \text { for some } n \text {. }
$$

Our aim in this case is slightly different, namely to construct an $n$-skeleton $B$ such that $B \vDash_{n} \overline{T H^{\prime}}\left(\varepsilon_{\beta}\right)$.

By 3.4(3) it is enough to construct an $n$-skeleton $B$ such that

(7) $\forall z<\min B B \vDash \forall x \exists y\left(H^{k}\right)_{\left(\varepsilon_{\underline{\beta}}\right)_{z}}(x)=y$ for every $k$ for which the sentence $\forall z \forall x \exists y\left(H^{k}\right)_{\left(\varepsilon_{\underline{\beta}}\right)_{z}}(x)=y$ is less than $n$.

Let $a=n^{c}$, where - let us remind $-c$ is the constant from Lemma 4.2. Arguing as in the previous case we obtain an $\varepsilon_{\beta}$-large set $A$ such that the set $B=h_{3}[A]$ minus last $a$ elements is a $2_{3}^{x}$-scattered $n^{c}$-skeleton.

Let $a_{0}=\min A, b_{0}=\min B$. Obviously $b_{0} \leq a_{0}-1$. We show that $A$ is $\left(\varepsilon_{\beta}\right)_{b_{0}}+5$-large. It is enough to show that

$$
\left(\varepsilon_{\beta}\right)_{b_{0}+1} \Rightarrow \leq 3\left(\varepsilon_{\beta}\right)_{b_{0}}+5 .
$$

If $\beta$ is nonlimit the proof is the same as the proof of $\omega^{\varepsilon_{\gamma}+1} \Rightarrow_{\leq 3} \varepsilon_{\gamma}+5$. If $\beta$ is limit then $\left(\varepsilon_{\beta}\right)_{b_{0}+1} \Rightarrow_{0} \varepsilon_{\gamma+1}$, where $\left(\varepsilon_{\gamma}\right)=\left(\varepsilon_{\beta}\right)_{b_{0}}$ and since $\varepsilon_{\gamma+1} \Rightarrow_{1} \omega^{\varepsilon_{\gamma}+1}$, (8) follows.

Thus by (6) the set $h_{3}[A]$ is $\left(\varepsilon_{\beta}\right)_{b_{0}}+5$-large and hence $B$ is $\left(\varepsilon_{\beta}\right)_{\beta_{0}}+4$ large.

Now by a reasoning similar to that in 4.2 we show that

$B \vDash \forall x \exists y\left(H^{k}\right)_{\left(\varepsilon_{\underline{\beta}}\right)_{b_{0}}}(x)=y$ for every $k$ such that

$$
\left\ulcorner\forall x \exists y H^{k}(x)=y\right\urcorner<n .
$$

Take $x, z \leq b_{0}$. Since $\left(\varepsilon_{\beta}\right)_{b_{0}} \Rightarrow_{0}\left(\varepsilon_{\beta}\right)_{z}$, it follows that $y^{\prime}=\bar{F}_{\left(\varepsilon_{\beta}\right)_{z}}(x) \leq$ $\bar{F}_{\left(\varepsilon_{\beta}\right)_{b_{0}}}(x) \leq b_{\varepsilon_{\beta}+2}$. The estimation of the witness of $y^{\prime}=\bar{F}_{\left(\varepsilon_{\beta}\right)_{z}}(x)$ is essentially the same as the estimation of the witness of $y=\bar{F}_{\left(\varepsilon_{\beta}\right)_{b_{0}}}(x)$ and we obtain the same upper bound $b_{\varepsilon_{\beta}+3}$, which shows $(7)$. This finishes the proof of $1.14(\Rightarrow)$.

\section{References}

[1] G. Gentzen, Beweisbarkeit und Unbeweisbarkeit von Anfangsfählen der transfiniten Induktion in der reinen Zahlentheorie, Math. Ann. 119 (1943), 140-161.

[2] P. Hájek and J. Paris, Combinatorial principles concerning approximations of functions, Arch. Math. Logik Grundlag. 26 (1987), 13-28.

[3] J. Ketonen and R. Solovay, Rapidly growing Ramsey functions, Ann. of Math. 113 (1981), 267-314.

[4] H. Kotlarski and Z. Ratajczyk, Inductive full satisfaction classes, Ann. Pure Appl. Logic 47 (1990), 199-223. 
[5] K. McAloon, Paris-Harrington incompleteness and progressions of theories, in: Proc. Sympos. Pure Math. 42, Amer. Math. Soc., 1985, 447-460.

[6] J. Paris and L. Harrington, A mathematical incompleteness in Peano arithmetic, in: Handbook of Mathematical Logic, North-Holland, 1977, 1133-1142.

[7] Z. Ratajczyk, A combinatorial analysis of functions provably recursive in $I \Sigma_{n}$, Fund. Math. 130 (1988), 191-213.

[8] - Subsystems of the true arithmetic and hierarchies of functions, Ann. Pure Appl. Logic, to appear.

[9] U.Schmerl, A fine structure generated by reflection formulas over primitive recursive arithmetic, in: Logic Colloquium 78, M. Boffa, K. McAloon and D. van Dalen (eds.), North-Holland, Amsterdam 1979, 335-350.

[10] D. Schmidt, Built-up systems of fundamental sequences and hierarchies of numbertheoretic functions, Arch. Math. Logik Grundlag. 18 (1976), 47-53.

[11] S. Wainer, Ordinal recursion, and a refinement of the extended Grzegorczyk hierarchy, J. Symbolic Logic 37 (1972), 281-292.

INSTITUTE OF MATHEMATICS

WARSAW UNIVERSITY

BANACHA 2

00-913 WARSZAWA 59, POLAND

Received 15 October 1990;

in revised form 26 September 1991 